\title{
Experimental evidence of thermal fluctuations on the X-ray absorption near-edge structure at the aluminum $K$-edge
}

\author{
D. Manuel, ${ }^{1, *}$ D. Cabaret,${ }^{1}$ Ch. Brouder ${ }^{1}$ Ph. Sainctavit, ${ }^{1}$ A. Bordage,${ }^{2}$ and N. Trcera ${ }^{3}$ \\ ${ }^{1}$ Université Pierre et Marie Curie (UPMC), IMPMC, \\ UMR CNRS 7590, 4 place Jussieu, 75252 Paris Cedex 05, France \\ ${ }^{2}$ Wigner Research Centre for Physics, Hungarian Academy of Sciences H-1525 Budapest, P.O.B. 49., Hungary \\ ${ }^{3}$ Synchrotron SOLEIL, BP 48, 91192 Gif sur Yvette, France
}

(Dated: March 22, 2012)

\begin{abstract}
After a review of temperature-dependent experimental x-ray absorption near-edge structure (XANES) and related theoretical developments, we present the Al $K$-edge XANES spectra of corundum and beryl for temperature ranging from $300 \mathrm{~K}$ to $930 \mathrm{~K}$. These experimental results provide a first evidence of the role of thermal fluctuation in XANES at the Al $K$-edge especially in the preedge region. The study is carried out by polarized XANES measurements of single crystals. For any orientation of the sample with respect to the x-ray beam, the pre-edge peak grows and shifts to lower energy with temperature. In addition temperature induces modifications in the position and intensities of the main XANES features. First-principles DFT calculations are performed for both compounds. They show that the pre-edge peak originates from forbidden $1 s \rightarrow 3 s$ transitions induced by vibrations. Three existing theoretical models are used to take vibrations into account in the absorption cross section calculations: i) an average of the XANES spectra over the thermal displacements of the absorbing atom around its equilibrium position, ii) a method based on the crude Born-Oppenheimer approximation where only the initial state is averaged over thermal displacements, iii) a convolution of the spectra obtained for the atoms at the equilibrium positions with an approximate phonon spectral function. The theoretical spectra so obtained permit to qualitatively understand the origin of the spectral modifications induced by temperature. However the correct treatment of thermal fluctuation in XANES spectroscopy requires more sophisticated theoretical tools.
\end{abstract}

\section{INTRODUCTION AND STATE OF THE ART}

$\mathrm{X}$ ray absorption near-edge structure (XANES) spectroscopy is a powerful technique to probe the empty states in solids and to determine the local structure around a selected atom. The interpretation of $K$ edge XANES spectra is not straightforward and often requires the use of simulation tools, which are traditionally based on the density functional theory (DFT). XANES calculations for inorganic solids usually consider the atoms at fixed positions, even if in reality atoms are subjected to quantum thermal fluctuations that reduce to the zero point motion at $T=0 \mathrm{~K}$. Recently, it has been theoretically shown that vibrations could have a spectacular effect at the $\mathrm{Al} K$-edge, allowing $1 s$ to $3 \mathrm{~s}$ dipole transitions. $\stackrel{1}{*}$ Such forbidden transitions were previously invoked to be responsible for the pre-edge feature that occurs at the $\mathrm{Al} K$-edge in several alumino-silicate minerals ${ }^{2}$ and at the Si $K$-edge in silicon diphosphate $\underline{3}^{3}$ Moreover, Ankudinov and Rehr stated that atomic displacements of order of typical Debye-Waller factors could reveal some forbidden transitions. $\stackrel{4}{ }$ Consequently, the fact that vibrations may be able to induce additional peaks (in the pre-edge or at higher energies) suggests that XANES could be considered as a relevant probe of quantum thermal fluctuations. It is already the case in organic molecules, the vibronic fine structure of which can be observed in XANES or NEXAFS (Near-Edge Xray Absorption Fine Structure) spectra. For instance in the last ten years, XANES spectroscopy has revealed the vibronic structure of naphtalene, ${ }^{5}$ bipheny ${ }^{6}$ and even larger molecules such as $\mathrm{NTCDA}^{7}$ at the $\mathrm{C} K$-edge, the vibronic structure of acetonitrile ${ }^{8}$ and acrylonitrile ${ }^{9}$ at the N $K$-edge, and more recently the one of halogenated acenaphthenequinone ${ }^{10}$ at the $\mathrm{O} K$-edge. The vibronic component of the lowest core-excited state of $\mathrm{OH}$ and OD was observed at the O $K$-edge as well. 11

In this paper, we focus on the thermal effects in two inorganic crystalline solids at the $\mathrm{Al} K$-edge: corundum $\left(\alpha-\mathrm{Al}_{2} \mathrm{O}_{3}\right)$ and beryl $\left(\mathrm{Be}_{3} \mathrm{Al}_{2} \mathrm{Si}_{6} \mathrm{O}_{18}\right)$. Polarized XANES spectra have been measured on single-crystals for temperatures ranging from $300 \mathrm{~K}$ to $930 \mathrm{~K}$. Our purpose is to show that thermal fluctuations induce substantial spectral modifications, especially in the pre-edge region. Very few studies were carried out in inorganic solids towards the same goal. As far as we know, thermal effects measured in XANES spectroscopy mainly concern the Ti $K$ pre-edge region in oxides. Such a temperature dependence was first observed by Durmeyer et al $\frac{12}{12}$ in $\mathrm{TiO}_{2}$ (rutile), $\mathrm{Li}_{4 / 3} \mathrm{Ti}_{5 / 3} \mathrm{O}_{4}$ and $\mathrm{LiTi}_{2} \mathrm{O}_{4}$. The main pre-edge peak was found to grow with temperature, and to slightly shift to lower energy for the first two compounds. Later, the temperature dependence of the polarized pre-edge structure at the $\mathrm{Ti} K$-edge in rutile was measured at low and room temperature 13 Thermal effects were essentially visible through an increase of intensity of the first two pre-edge peaks. The temperature dependence of the electric dipole and quadrupole contributions of the Ti $K$ edge in rutile was investigated in the $6 \mathrm{~K}-698 \mathrm{~K}$ range by Collins and Dmitrienko in 2010 at the Diamond Light 
Source $\stackrel{14}{\underline{1}}$ Nozawa et al $\stackrel{15}{\underline{1}}$ measured the temperature dependence of the Ti $K$-edge XANES in $\mathrm{SrTiO}_{3}$ from $15 \mathrm{~K}$ to $300 \mathrm{~K}$, showing an increase of the first two pre-edge peaks along with a slight shift to lower energy of the second pre-edge peak and of the absorption edge. These effects were attributed to random thermal vibrations of $\mathrm{Ti} \cdot \frac{15}{1}$

Apart from these last examples at the Ti $K$-edge, most of temperature-dependent XANES measurements were carried out in order to study phase transition mecanisms or to determine the local structure below and above the transition temperature. For instance in some perovskite crystals, significant spectral changes in the $K$ pre-edge region are induced by temperature, and are interpreted in relation to phase transition. The literature reports temperature-dependent x-ray absorption spectra (including pre-edge) in perovskite titanates at the Ti $K$-edge,,$\frac{16-21}{2}$ in perovskite manganites at the Mn $K$-edge $\stackrel{22,23}{2}$ and at the $\mathrm{O} K$-edge $\stackrel{24,25}{=}$ in zirconates at the $\mathrm{Zr} K$-edge, $\stackrel{26}{r}$ niobiates at the $\mathrm{Nb} K$-edge, $27-31$ in the $\mathrm{La}_{1-x} \mathrm{Sr}_{x} \mathrm{FeO}_{3-\delta}$ system at the $\mathrm{Fe} K$-edge, ${ }^{32}$ in a $\mathrm{La}(\mathrm{Fe}, \mathrm{Ni}) \mathrm{O}_{3}$ solid oxide fuel cell cathode at the $\mathrm{O} K$-edge,, 33 and more recently in the $\operatorname{Pr}_{0.5} \mathrm{Ca}_{0.5} \mathrm{CoO}_{3}$ cobaltite at the Ca $L_{2,3}$-edges and the $\operatorname{Pr} L_{3}$ and $M_{4,5^{-}}$ edges ${ }^{34}$ Phase transition studies using temperaturedependent XANES are not restricted to the perovskite structure. In particular $\mathrm{Cu} K$-edge and La $L$-edges spectra in $\mathrm{La}_{2-x} \mathrm{Sr}_{x} \mathrm{CuO}_{4}$ cuprates were recorded at room temperature and at $78 \mathrm{~K} \cdot \frac{35}{4} \mathrm{An}$ angular dependence XANES study was performed at the $\mathrm{V} K$-edge in $\mathrm{VO}_{2}$ for temperatures lower and higher than the metal-insulator transition temperature at $68^{\circ} \mathrm{C} \underline{36}$ Several studies reported in literature deal with oxide glass and melt structures. For instance, the local structural environment of $\mathrm{Ti}$ in Na-, K-, and Ca-titanosilicate glasses and melts was determined by Ti $K$-edge x-ray absorption spectroscopy at temperatures ranging from 293-1650 K $\stackrel{37}{=}$ The $\mathrm{Zr} K$ edge in a $\mathrm{ZrO}_{2}-\mathrm{MgO}-\mathrm{Al}_{2} \mathrm{O}_{3}-\mathrm{SiO}_{2}$ glass was measured for various temperatures around $T_{g}(1085 \mathrm{~K}) \stackrel{38}{3}$ The crystalline and melt structures of $\mathrm{Al}_{2} \mathrm{O}_{3}$ and $\mathrm{MgAl}_{2} \mathrm{O}_{4}$ were investigated using temperature dependent XANES spectroscopy at the $\mathrm{Al}$ and $\mathrm{Mg} K$-edges $\frac{39}{} \mathrm{~A}$ similar study concerns the crystal, glass and melt structures in the CaO-MgO- $\mathrm{Al}_{2} \mathrm{O}_{3}-\mathrm{SiO}_{2}$ system at the $\mathrm{Al}, \mathrm{Si}$ and $\mathrm{Ca} K-$ edges $\stackrel{40}{-2}$ Again at the $\mathrm{Si} K$-edge, the structure of $\mathrm{SiO}_{2}$ polymorphs were studied using XANES spectroscopy from room temperature to $2030 \mathrm{~K}$, above the melting point at $2000 \mathrm{~K} \stackrel{41}{=}$ Furthermore, at the oxygen $K$-edge in liquid water, variations were observed between the room temperature spectrum and the $90^{\circ} \mathrm{C}$ one and found to be related to a change of local environment of a significant amount of molecules $\stackrel{42}{\underline{4}}$

Temperature dependent x-ray absorption spectroscopy was also used to study chemical reactions. For instance, in catalysis, the local structure of $\mathrm{Al}$ in zeolites was examined to understand the processes that take place during steam activation of these complex cagelike porous compounds. Hence $\mathrm{Al} K$-edge measurements in several zeolites were carried out in the range $300 \mathrm{~K}-975 \mathrm{~K}$, in vacuum or in a flow of helium saturated with water, $\underline{43}-46$ and in other working conditions of the catalysis. $\stackrel{47}{ } \mathrm{In}$ Refs. 43, 44, 46, and 47 a pre-edge peak appears when temperature is increased. The presence of this Al $K$ preedge peak at high temperature is interpreted as the signature of three-fold coordinated $\mathrm{Al}$ in the zeolite structure. Other temperature effects are observable at higher energies in the XANES region, and attributed to the transformation of a given amount of octahedrally coordinated $\mathrm{Al}$ to tetrahedrally coordinated $\mathrm{Al} \stackrel{44,45}{\underline{4}}$ Recently, in a totally different field, the temperature dependence of $\mathrm{Fe} K$-edge XANES spectra of $\mathrm{FePt} / \mathrm{Fe}_{3} \mathrm{O}_{4}$ nanoparticles has been measured from $300 \mathrm{~K}$ to $870 \mathrm{~K}$ in order to understand the annealing process, which enables the building of magnetic nanocomposites with combined magnetic properties $\underline{48}$ A strong temperature dependence was observed in $\mathrm{Fe} L_{2,3}$-edges spectra of Fe impurities in $\mathrm{MgO}$ thin films in the range $77 \mathrm{~K}-500 \mathrm{~K}$; it was attributed to the thermal population of low-lying Fe $3 d$ excited states, that are present due to the spin-orbit coupling $\underline{\underline{49}}$ At last, the valence state of $\mathrm{Yb}$ in $\mathrm{YbC}_{2}$, investigated by $\mathrm{Yb} L_{3}$ $\mathrm{XANES}$ spectroscopy at low and high temperatures, is found to be stable from $15 \mathrm{~K}$ to $1123 \mathrm{~K}$, so providing new information about this long-known compound, $\underline{\underline{50}}$

In all the references cited in the previous two paragraphs, the role of nuclear motion in the temperaturedependent XANES spectra was not investigated. A thorough study of thermal fluctuations could then bring new insights in the interpretation of the temperaturedependent spectral features whatever the purpose of the experiments is (catalysis, phase transition, etc.).

The modeling of nuclear motion in the absorption cross section of solids is a challenging task. In particular, the presence of phonon modes in crystalline solids generates a dynamic disorder, which has a completely different behavior from the static disorder that can be found in glasses. Far from the edge, in the extended $\mathrm{x}$ ray absorption fine structure (EXAFS) region, vibrations are taken into account through a Debye-Waller factor $\exp \left(-2 k^{2} \sigma^{2}\right) \stackrel{51,52}{5}$ Since this factor vanishes for $k \simeq 0$, it cannot reproduce well the thermal effects in the XANES region and especially in the pre-edge region. Fujikawa and co-workers showed in a series of papers of increasing sophistication $\underline{53}-\underline{55}$ that the treatment of vibrations could be achieved by the convolution of the "phononless" x-ray absorption spectrum with the phonon spectral function. To our knowledge, this theoretical work has not been applied to any real case yet. A basic and simple idea to take vibrations into account is to calculate the absorption cross-section for a configuration where the absorbing atom is shifted from its equilibrium position $\stackrel{4.26}{=}$ For instance, at the $\mathrm{B} K$-edge of $\mathrm{ABO}_{3}$ perovskite structures, the approach used by Vedrinskii et al. ${ }^{26}$ to treat the temperature effects is based on the assumption that the area of the pre-edge peak is proportional to the mean square displacements of the $\mathrm{B}$ atom along the O-B-O chain. In such a way, the symmetry-breaking of the absorbing atom 
site generates or increases local $p$ - $d$ and $p$ - $s$ hybridization. Brouder and co-workers ${ }^{1,56}$ developed a different approach assuming that vibrational energies are small with respect to the instrumental resolution, and using the crude Born-Oppenheimer approximation so that only core-hole motion remains. The resulting expression for the absorption cross section shows that, at the $K$ edge, vibrations enable electric-dipole transitions to $3 s$ and $3 d$ final states, that are not due to local hybridization with the $p$ states. In the case of organic molecules, more advanced theories are employed to reproduce the vibronic structure observed in XANES spectra. Beyond the BornOppenheimer approximation, a vibronic coupling theory was developed and successfully applied to the C$1 \mathrm{~s}$ absorption spectra of ethylene $\left(\mathrm{C}_{2} \mathrm{H}_{4}\right)^{57}$ and ethyne $\left(\mathrm{C}_{2} \mathrm{H}_{2}\right)^{58}$ and isotopomers. Besides, many approaches calculate Franck-Condon factors based on a vibrational eigenmode analysis in the ground and excited states. In particular, the linear coupling model was used to compute the Franck-Condon factors for NEXAFS spectra at the $\mathrm{O} K$-edge in formaldehyde,, 59 at the $\mathrm{C} K$-edge in formaldehyde, 60 naphtalene ${ }^{5}$, gaseous cyclopropane,$\underline{61}$ and acetic acid, ${ }^{62}$ at the $\mathrm{N} K$-edge in gaseous pyridine, ${ }^{63}$ acetonitrile ${ }^{8}$ and acrylonitrile. $\frac{9}{}$ In Ref. 9 a direct calculation of the Franck-Condon amplitudes is also performed. However, the Franck-Condon approximation ignores the impact of nuclear motion on the electronic transition amplitude. To first order, this impact is referred to as the Herzberg-Teller effect ${ }^{64}$ To go beyond the FranckCondon approximation, the nuclear degrees of freedom of molecules was modeled using various molecular dynamics (MD) techniques. In doing so, atomic configurations are generated at finite temperature and then used as input in cross-section calculations. Finally, the individual spectra associated to each sampled atomic configuration are averaged over. Using this methodology, vibrations were included in XANES calculations at the N $K$-edge of several prototype molecules: upon classical MD sampling, ${ }^{65-67}$ and upon path-integral molecular dynamics (PIMD) 66.68 PIMD sampling enables to consider the quantum nature of nuclear motion, which has been found to be of noticeable importance to accurately simulate XANES of $\mathrm{N}_{2},{ }^{66}$, and s-triazine and glycine ${ }^{68}$ The sensitivity of photoabsorption spectroscopy to a quantum treatment of nuclear motion was also highlighted in the optical range ${ }^{69}$ and in the UV range ${ }^{70}$ The MD methodology is not restricted to organic molecules; simulations of the density and temperature dependence of XANES in warm dense aluminum plasmas were achieved using ab initio ${ }^{71,72}$ and classical ${ }^{73} \mathrm{MD}$ and recently they have been compared to experiments $\underset{74,75}{ }$

In the present study, DFT calculations based on planewave formalism are performed in order to understand the spectral modifications induced by temperature that are observed experimentally. Reference theoretical Al $K$ XANES spectra of corundum and beryl are first obtained considering the atoms at their equilibrium positions. Then three existing methods are used to account for vibrations: (i) a calculation considering thermal displacements of the absorbing atom around its equilibrium position, (ii) the method developed by Brouder et al., 1 (iii) a convolution of the spectra obtained for the atoms at the equilibrium positions with an approximate phonon spectral function.

The paper is organized as follows. Section III is dedicated to the methods. First the temperature-dependent XANES experiments at the $\mathrm{Al} K$-edge of corundum and beryl are described, with a specific attention paid to the self-absorption correction procedure. Second, the three theoretical DFT methods used to take the vibrations into account are detailed. Section III is devoted to the description of the experimental spectra recorded for temperatures ranging from $300 \mathrm{~K}$ to $930 \mathrm{~K}$. In section IV the experimental results are analyzed with the help of the DFT calculations, and discussed in the context of the temperature-dependent XANES spectra already reported in the literature. The conclusion of this work is given in Section $\mathrm{V}$

\section{METHODS}

\section{A. Experimental setup}

Two single-crystalline samples containing aluminum in 6 -fold coordination with oxygen are studied. The first is a cylindrical synthetic transparent ruby $\left(\alpha-\mathrm{Al}_{2} \mathrm{O}_{3}\right.$ with $18 \mathrm{ppm}$ of $\mathrm{Cr}$ and $10 \mathrm{ppm}$ of $\mathrm{Ti}$ ) of $4.0 \mathrm{~mm}$ diameter and $1.0 \mathrm{~mm}$ thickness, which will thereafter be denoted as corundum. The second is a parallelepipedic section of a natural green emerald from Colombia $\left(\mathrm{Be}_{3} \mathrm{Al}_{2} \mathrm{Si}_{6} \mathrm{O}_{18}\right.$ with $238 \mathrm{ppm}$ of $\mathrm{Fe}, 78 \mathrm{ppm}$ of $\mathrm{Cr}, 59 \mathrm{ppm}$ of $\mathrm{Sc}$ and $36 \mathrm{ppm}$ of $\mathrm{Co}$ ) of $10.0 \mathrm{~mm}$ length, $3.6 \mathrm{~mm}$ width and $1.2 \mathrm{~mm}$ thickness, which will be denoted as beryl. The samples were analyzed using the CAMPARIS electronic microprobe at Université Pierre et Marie Curie - Paris 6, France.

X-ray absorption experiments were performed at LUCIA beamline in the French synchrotron facility SOLEIL 76 The synchrotron was operating in the top-up mode at $2.75 \mathrm{GeV}$ with a current of $400 \mathrm{~mA}$. The beam spot size was set to $1 \times 2 \mathrm{~mm}^{2}$ and the energy range chosen to include the $\mathrm{Al} K$-edge $(1559.6 \mathrm{eV} \text { in bulk })^{77}$ was set from $1550 \mathrm{eV}$ to $1700 \mathrm{eV}$, hence measuring pre-edge, XANES and the beginning of EXAFS. Energy selection was performed through the combination of an HU52 "Apple II" type undulator and a double KTP (011) crystal monochromator. The pressure in the experimental chamber was $10^{-5}$ mbar.

The samples were held between a parallelepipedic boron nitride furnace and a punched molybdenum lamella fixed to the furnace, allowing temperatures ranging from $300 \mathrm{~K}$ to $950 \mathrm{~K}$. This holder was fixed on a mobile stage, allowing translations along a cartesian $x y z$ coordinate system plus a $z$-axis rotation, where the $x$-axis corresponds to the beam direction and the $y$-axis to the 
(horizontal) linear polarization $\varepsilon$ of the beam. In order to minimize self-absorption effects, the sample surfaces should be orthogonal to the beam axis. For fluorescence detection, the sample holder has been slightly rotated by $15^{\circ}$ around $z$-axis.

Corundum and beryl point groups are $\overline{3} \mathrm{~m}$ and $\frac{6}{\mathrm{~m}} \mathrm{~mm}$ respectively ${ }^{78,79}$ This implies that both materials are dichroic in the electric dipole approximation. ${ }^{80}$ The single-crystals were set on the sample holder in order to measure successively $\sigma_{\|}$and $\sigma_{\perp}$ spectra, corresponding respectively to $\varepsilon$ parallel and perpendicular to the high symmetry axis, i.e. the three-fold symmetry axis for corundum and the six-fold symmetry axis for beryl.

Total fluorescence yield was measured by a four element silicon drift diode (SDD) detector with a total active area of $40 \mathrm{~mm}^{2}$ protected from infrared and visible radiations by a thin beryllium window. In order to maximize the signal/noise ratio, each point was obtained after a six second acquisition time and five consecutive spectra were measured for each configuration (a configuration consists of a sample, an orientation and a temperature). This system produces five intensity outputs, one for each of the four $\operatorname{SDD}\left(I_{1}, I_{2}, I_{3}, I_{4}\right)$ and one for the incident beam $\left(I_{0}\right)$, measured before the sample. For each spectrum, each $I_{j}$ was then divided by $I_{0}$, followed with a normalization on the 1590-1700 eV energy range (spectra are divided by their mean values on this region) giving $\left(I_{j} / I_{0}\right)_{\text {norm }}$. Then, $\left(I_{j} / I_{0}\right)_{\text {av }}$ was calculated for each configuration by averaging five consecutive acquisitions of $\left(I_{j} / I_{0}\right)_{\text {norm }}$. Since self-absorption effects are angle dependent and since the four SDD were not at the exact same spatial position, the four $\left(I_{j} / I_{0}\right)_{\text {av }}$ were not directly comparable. Self-absorption correction was performed using the formula: 81,82

$$
\sigma_{j}(\omega)=\frac{\mu_{j}(\omega)}{\mu_{j}\left(\omega_{0}\right)}=\frac{N_{j}(\omega)\left(\beta \frac{\sin \theta_{i}}{\sin \theta_{f, j}}+\gamma\right)}{\left(\beta \frac{\sin \theta_{i}}{\sin \theta_{f, j}}+\gamma+1\right)-N_{j}(\omega)}
$$

in which $\mu_{j}$ is the edge absorption coefficient for detector $j, N_{j}(\omega)=\frac{\left(I_{j} / I_{0}\right)_{\mathrm{av}}(\omega)}{\left(I_{j} / I_{0}\right)_{\mathrm{av}}\left(\omega_{0}\right)}$ is the measured intensity on silicon drift diode $j$, normalized at a fixed pulsation $\omega_{0}$ (chosen after the main edge at $\hbar \omega_{0}=1653 \mathrm{eV}$ ), $\theta_{i}=75^{\circ}$ is the angle between the beam axis and the sample surface, $\theta_{f, j}$ is the angle between the sample surface and the outgoing fluorescence beam (which depends on each detector $j$ position). Coefficient $\beta$ is equal to $\frac{\mu_{\text {tot }}\left(\omega_{\text {fluo }}\right)}{\mu\left(\omega_{0}\right)}$ where $\omega_{\text {fluo }}$ is the $K \alpha_{1}$-fluorescence pulsation $\left(\hbar \omega_{\text {fluo }}=1486.7 \mathrm{eV}\right)$ and $\mu_{\text {tot }}$ is the total absorption coefficient, including edge absorption $\mu$ and background contribution $\mu_{\mathrm{bg}}$ of other atomic species in the sample. Finally $\gamma$ is equal to $\frac{\mu_{\mathrm{bg}}}{\mu}\left(\omega_{0}\right)$. Values for $\beta$ and $\gamma$ are calculated based on crystal stoichiometry, atomic masses, unit cell geometry and using experimental tabulated atomic values. ${ }^{83}$ For example, $\mu_{\text {tot }}(\omega)=\sum_{\text {at }} \rho_{\text {at }} \mu_{\text {at }}^{\text {table }}(\omega) / M_{\text {at }}$ with $\rho_{\text {at }}=N_{\text {at }} M_{\text {at }} \mathcal{N}_{A} / V_{\text {u.c. }}$ where the sum is over all the atoms in the unit cell and for each atom $\mu_{\mathrm{at}}^{\text {table }}$ is the tabulated value in Barns/atom, $M_{\text {at }}$ is the molar mass in g.mol ${ }^{-1}, N_{\text {at }}$ is the number of atoms 'at' in the unit cell, $V_{\text {u.c. }}$ is the unit cell volume in $\mathrm{cm}^{3}$ and $\mathcal{N}_{A}$ is the Avogadro constant. Such a correction gives comparable values of $\sigma_{j}$ for each detector, each one being normalized at the point of energy $\hbar \omega_{0}$. By using such a procedure to perform self-absorption correction, averaging over the four detectors is possible and gives the spectra $\sigma_{\|}$and $\sigma_{\perp}$ shown in section [II No smoothing were applied to the spectra.

\section{B. Theoretical methods}

In order to explain the experimental spectral features observed when temperature is increased, first-principles calculations were performed in the density functional theory framework using the Quantum-Espresso suite of codes ${ }^{84}$ The $1 s$ core-hole effects were taken into account within a $2 \times 2 \times 2$ trigonal supercell for corundum, containing 80 atoms,,$\frac{78}{5}$ and a $2 \times 1 \times 1$ hexagonal supercell for beryl, containing 58 atoms $\stackrel{79}{ }$ Troullier-Martins $\$ 55$ normconserving pseudopotential were used. The pseudopotentials of $\mathrm{Al}$ and $\mathrm{Si}$ were generated using the $3 s, 3 p$ and $3 d$ orbitals as valence states, with 2.00 Bohr cutoff radii and considering the $d$ states as local. The pseudopotential of the $\mathrm{Al}$ absorbing atom was generated with only one $1 \mathrm{~s}$ electron in the $\mathrm{Al}$ electronic configuration. The oxygen and beryllium pseudopotentials were built using the $2 s$ and $2 p$ as valence states (local part $p$ ) with cutoff radii of 1.46 Bohr for O and of 2.00 Bohr for Be. Self-consistent charge densities were calculated in the generalized gradient approximation of Ref 86, using the PWscf code, at the $\Gamma$ point, with plane-wave energy cut-off of $80 \mathrm{Ry}$. Thereafter, local and partial density of states were calculated using Löwdin projections on a $4 \times 4 \times 4 k$-point grid with a Gaussian broadening parameter of $0.3 \mathrm{eV}$. XANES cross section calculations were performed using the XSpectra code. $\stackrel{87.88}{ }$ The all-electron wave-function of the Al absorbing atom was reconstructed using the Projector Augmented Wave method, $\stackrel{89}{ }$ with augmentation region radii of 2.0 Bohr. The spectra were computed on $4 \times 4 \times 4 k$-point grid with a broadening parameter $\gamma=0.6 \mathrm{eV}$.

Three methods were used to take into account the effects of vibrations without having to calculate phonon modes. First, adapting the idea of Ref 15 on vibrations, only the absorbing atom was moved in the crystal structure with respect to its equilibrium position. This method will be referred to as 'method 1' in the following section. As vibrations are concerned, spectra for several absorbing atom displacements $\mathbf{R}$ were calculated and averaged with a weighting core displacement distribution function $\rho(\mathbf{R})$. Within the harmonic approximation, this distribution function is linked to the thermal parameter matrix $\left[U_{i j}\right]$ through $\rho(\mathbf{R})=\exp \left(-\mathbf{R} \cdot \frac{U^{-1}}{2} \cdot \mathbf{R}\right)$. The components of the rank- 2 tensor $U$ are given by X-ray or neutron scattering measurements. In this approximation each spectrum is calculated with a static off-center ab- 


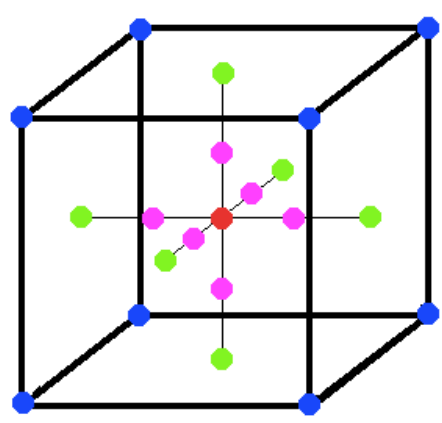

FIG. 1. A sketch of the 21-point cubic grid used in Eq. 2

sorbing atom, creating hybridization between $\mathrm{Al}$ valence orbitals and neighboring atoms orbitals, thus modifying valence states of the crystal. Vibration modeling comes from averaging spectra over a large number of absorbing atom displacements.

The absorbing atom motion was discretized on a cubic tridimensional grid consisting of 361 points. Equation 2 gives the cubic integral approximation on a grid of 21 points (see Fig. 11): $: \underline{=}$

$$
\begin{aligned}
\frac{1}{a^{3}} \int_{\text {cube }} f(\omega, \mathbf{R}) d \mathbf{R}= & \frac{1}{360}\left(-496 f_{m}+128 \sum f_{r}\right. \\
& \left.+8 \sum f_{f}+5 \sum f_{v}\right)+O\left(a^{6}\right),
\end{aligned}
$$

where $a$ is the length of the cube edge, $f(\omega, \mathbf{R})=\sigma_{e}(\omega, \mathbf{R}) \times \rho(\mathbf{R})$ is the weighted spectrum calculated with absorbing atom displaced of $\mathbf{R}$, $f_{m}=f(\omega, \mathbf{0})$ is the value at the center of the cube (red point), $\sum f_{r}$ is the sum of the values of $f$ at the 4 points halfway between the center of the cube and the center of each face (pink points), $\sum f_{f}$ is the sum of the values of $f$ at the center of each face (green points) and $\sum f_{d}$ is the sum of the values of $f$ at the vertices of the cube (blue points). The 361 point grid is an augmented $3 \times 3 \times 3$ version of the 21 point one. This discretization would lead to a $27 \times 21$ point grid, which reduces to only 361 points after avoiding double counting of similar points. The value of the integration cube edge $a$ is given by $2 \sqrt{U_{\text {eig }}}$, where $U_{\text {eig }}$ are the eigen values of the $U$ matrix. For the 'method 1' calculations, $a$ was chosen equal to 0.3 Bohr radius, in agreement with the thermal matrix parameters of corundum and beryl given in Refs. 79 and 91 .

The second method will be referred to as 'method 2,, ,, 56 Since vibrational energies are small with respect to the instrumental resolution, the closure approximation is made. In this approximation, the final vibrational states of the electrons+nuclei system are summed over. Hence atomic motions are assumed not to change the final electronic states that are calculated at the atomic equilibrium positions. Consequently, in 'method 2', only the position of the absorbing atom in the initial state is modified by vibrations. In practice, for a $K$-edge, the $1 s$ wave-function of the initial state is no more centered on the absorbing atom equilibrium position. This shifted $1 \mathrm{~s}$ wave-function is expanded over spherical harmonics centered on the equilibrium position. The $\ell=1$ component allows the transition to $3 \mathrm{~s}$ states. Contrary to 'method 1 ', this method does not create hybridization in the final state. Calculating absorption cross section in this method is based on Eq. (9) from Ref. 11. The $1 s$ wavefunction movements are calculated using the same core displacement distribution used in 'method 1', integrating over the same 361 point grid, but using a shorter value for $a(a=0.1$ Bohr radius as in Ref. 1).

The third method, denoted as 'method 3', is based on Fujikawa's work $\underline{54}$ Within the Franck-Condon approximation, the effect of vibrations on x-ray absorption spectroscopy can be represented as the convolution of the phonon spectral function with the x-ray absorption spectrum at equilibrium position. Here the phonon spectral function is approximated as a Gaussian distribution. Along with the two precedent methods, convoluted spectra are calculated. The convolution is performed between the first-principles equilibrium spectrum for a configuration and a Gaussian. The Gaussian equation is $1 /(\sqrt{2 \pi} \sigma) \exp \left(-x^{2} /(2 \sigma)^{2}\right)$, with parameter $\sigma$ being chosen for each configuration in order to reproduce the main edge intensity of method 1 spectra.

Calculated spectra presented in section IV are all normalized on the high-energy region, as was done for experimental data.

\section{EXPERIMENTAL RESULTS}

Figure 2 shows the self-absorption corrected XANES spectra of corundum and beryl recorded at different temperatures for both orientations. Spectra originating at $y=-0.5$ correspond to the difference $\sigma(T)-\sigma(300 \mathrm{~K})$. We observe here for the first time the slow evolution of the Al $K$ pre-edge feature with temperature. In addition to the expected increase in the peak intensity, $, 1,56$ a shift toward lower energy of the pre-edge structure is observed when temperature increases. Furthermore, this effect appears for each sample and for each orientation and seems to be general, as well as the decreasing intensities and broadening of main peaks with temperature.

Corundum $\sigma_{\|}$and $\sigma_{\perp}$ spectra are quite similar up to $1575 \mathrm{eV}$ : they both present a well-resolved pre-edge peak $\mathrm{P}$, the main peak $\mathrm{A}$ followed by the double feature $\mathrm{B}_{1}$ and $\mathrm{B}_{2}$. The $300 \mathrm{~K}$ polarized spectra are in agreement with those recorded in total electron yield shown in Ref. 92. As mentioned before, the intensity of peak $\mathrm{P}$ increases with temperature while its position is shifted towards lower energy. This is confirmed by the peak $\mathrm{p}$ in the difference spectra. Peak A also varies with temperature: it broadens, its intensity decreases and its position is shifted to lower energy (around $0.2 \mathrm{eV}$ and $0.3 \mathrm{eV}$ for $\sigma_{\|}$and $\sigma_{\perp}$ respectively). The decrease of peak A intensity corresponds to the difference spectra peak a and the shift to lower energy to a'. Peak a' is also related to the broadening of 

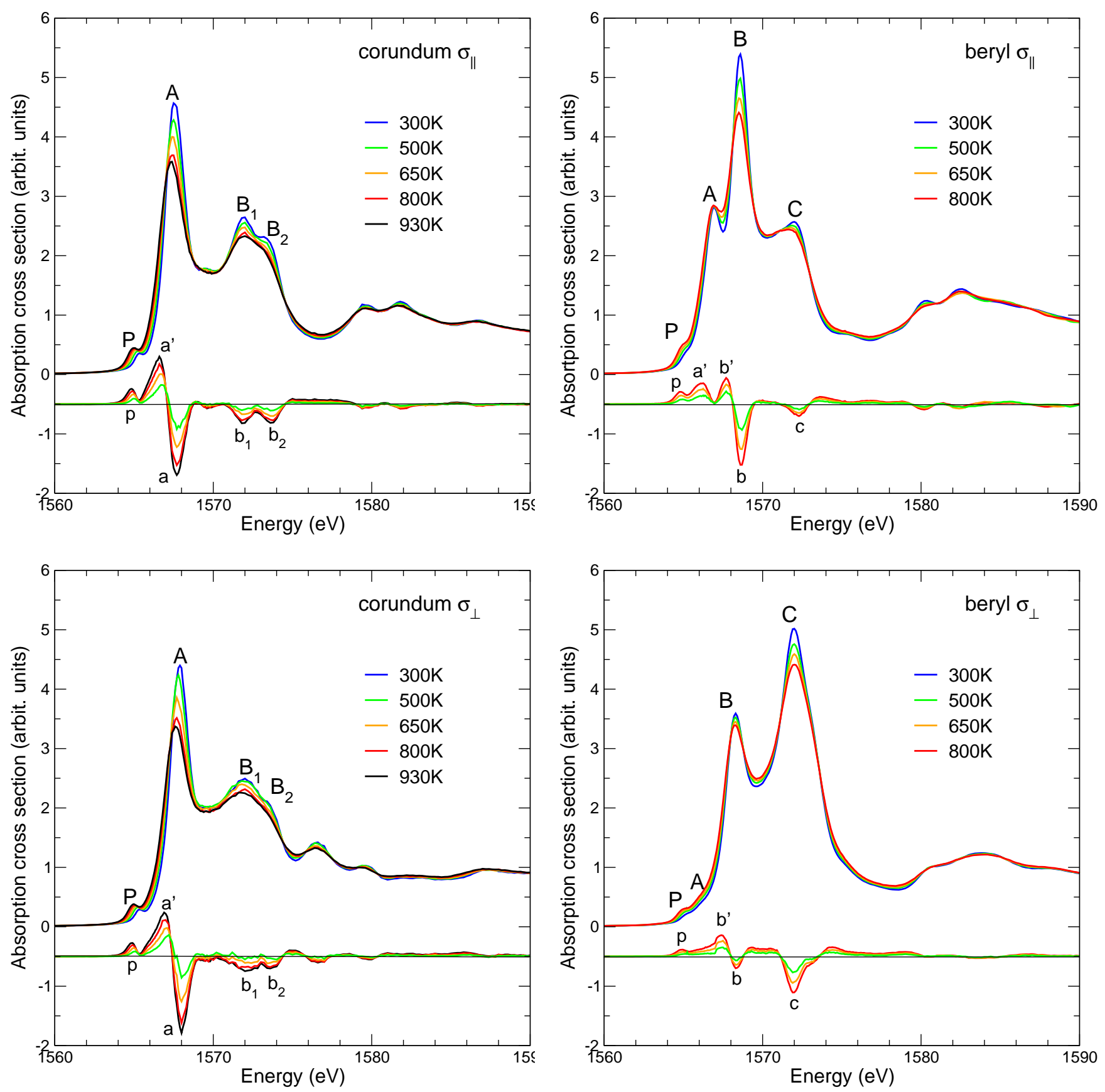

FIG. 2. Experimental X-ray absorption polarized spectra of corundum (left) and beryl (right) at the Al $K$-edge for different temperatures along with difference of each spectrum with respect to the $300 \mathrm{~K}$ reference. The top and bottom panels display the $\sigma_{\|}$and $\sigma_{\perp}$ spectra, respectively.

peak $A$ with temperature. Peaks $B_{1}$ and $B_{2}$ intensities also diminish and they only form one broad peak at $930 \mathrm{~K}$ in $\sigma_{\|}$and $\sigma_{\perp}$.

Beryl $\sigma_{\|}$and $\sigma_{\perp}$ spectra present much different shapes, unlike the case of corundum. In the energy range 1560$1575 \mathrm{eV}$, the $\sigma_{\|}$spectrum exhibits the pre-edge peak P and three main features A, B and C, feature B being around twice more intense than $\mathrm{A}$ and $\mathrm{C}$ at $300 \mathrm{~K}$. On the con- trary, the $\sigma_{\perp}$ spectrum exhibits two main features B and $\mathrm{C}$, preceded by a small shoulder $\mathrm{A}$ and the pre-edge $\mathrm{P}$. Peak C is more intense than peak B. Peak A and B of $\sigma_{\perp}$ are located at $0.7 \mathrm{eV}$ and $0.3 \mathrm{eV}$ lower than their corresponding peaks in $\sigma_{\|}$. The pre-edge peak P is less intense in $\sigma_{\perp}$ than in $\sigma_{\|}$at $300 \mathrm{~K}$ and its intensity increases more in $\sigma_{\|}$than in $\sigma_{\perp}$. Indeed the difference peak $\mathrm{p}$ appears better resolved and more intense in $\sigma_{\|}$than in $\sigma_{\perp}$. Peak 
A of $\sigma_{\|}$has a constant intensity with temperature but creates the difference peak a' due to broadening. The weak peak A of $\sigma_{\perp}$ decreases in intensity with temperature. The B peak intensity also decreases with temperature, as well as a slight shift of $0.1 \mathrm{eV}$ toward lower energy, that creates peaks $b$ and $b$ ' in the difference spectra, more pronounced in $\sigma_{\|}$than in $\sigma_{\perp}$. Peak $\mathrm{C}$ decreases and broadens with temperature, leading to feature $c$ in the difference spectra.
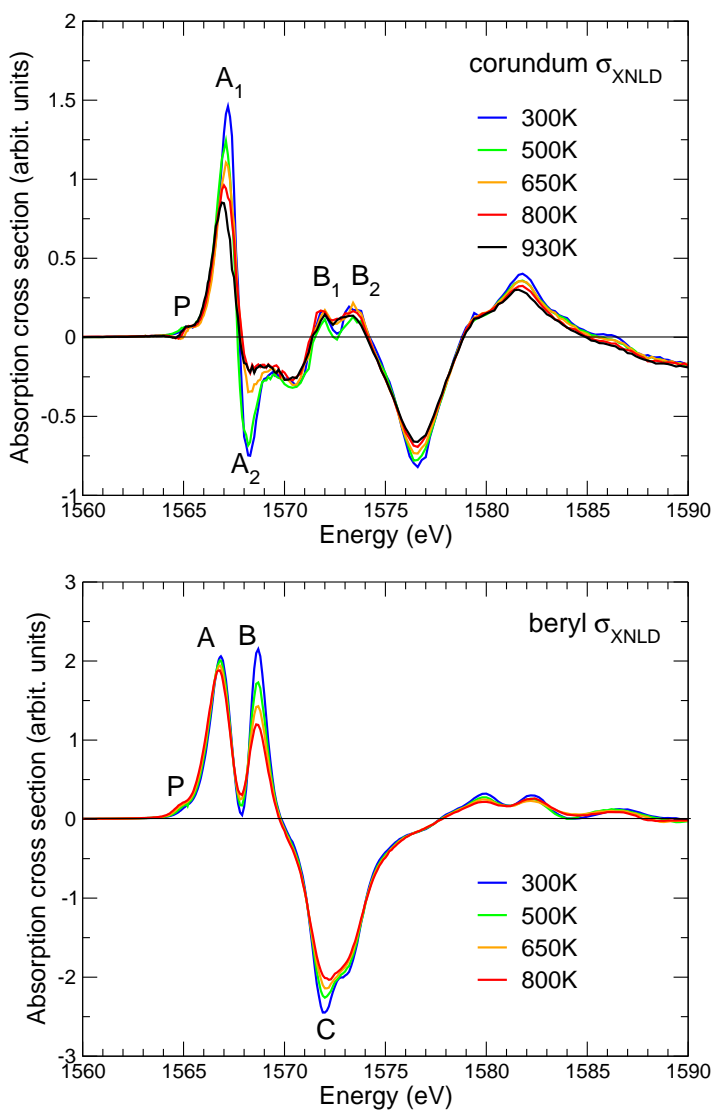

FIG. 3. Temperature dependence of the X-ray natural linear dichroism (XNLD) measured at the Al $K$-edge in corundum (top panel) and beryl (bottom panel). The cross section $\sigma_{\mathrm{XNLD}}$ is equal to the difference $\sigma_{\|}-\sigma_{\perp}$. Note that the $y$-axis range is twice larger for beryl than for corundum.

The x-ray natural linear dichroisms (XNLD) for the various experimental temperatures are plotted in Fig. 3 . The XNLD signals of the two samples are large and their variations with temperature do not change their general shape. XNLD on the pre-edge is quite small so that temperature dependence can hardly be seen. One mainly observes that some sharp XNLD maxima are reduced when the temperature increases: $\mathrm{A} 1$ and $\mathrm{A} 2$ peaks for corundum and B peak for beryl. One also notices that the rising edge and the maximum of the first XNLD feature is shifted to lower energies. This effect is observed for both corundum and beryl and can also be detected on the parallel and perpendicular cross-sections. It is apparently a key feature of the temperature evolution of the spectra. The features at high energies are only slightly modified. Sum rules relates XNLD to the electric quadrupole distribution of the empty DOS with $p$ symmetry on the aluminium site 93 The sum rules were first derived in the absence of electron-phonon coupling and it is not yet clear whether the sum rules still hold when temperature is present. A theoretical analysis of the temperature dependence of XNLD sum rules is beyond the scope of the present paper but would eventually give information on the modification of the electronic structure with the temperature.

In order to more quantitatively describe the behavior of the pre-edge peak, extraction was performed using $\mathrm{Fi}$ tyk software, ${ }^{94}$ which fits the main edge and pre-edge by the sum of an arctangent and a Gaussian. Figure 4 reports the fitted Gaussian intensities and center energies for each sample and each orientation. These quantitative values are in good agreement with the foregoing observations, a global increase of intensity with temperature is noticeable. While both orientations seem to progress similarly in corundum, it is not the case in beryl where $\sigma_{\perp}$ intensities grow less rapidly than in $\sigma_{\|}$. Concerning peak position, both compounds and orientations present a similar shift toward lower energies.

\section{INTERPRETATION AND DISCUSSION}

In this section, theoretical results are presented and discussed in order to try to understand the effects of temperature observed in the XANES spectra shown in Section III] First, a density of states (DOS) analysis is performed for both compounds showing the involvement of the $3 s$ empty states of the $\mathrm{Al}$ absorbing atom in the process of pre-edge peak creation. Then, theoretical spectra obtained by using the three different methods described in section II B are shown and compared together.

\section{A. DOS calculations: the nature of the pre-edge peak}

The pre-edge peak is not reproduced by purely electric dipole $1 s \rightarrow p$ transitions, as shown in the top panels of Fig. 5. Hence some transition toward non- $p$ states must be at stake. The bottom panels of Fig. 5 present partial and local density of states (DOS) of corundum and beryl. The $s, p$ and $d$ partial empty DOS are plotted for the absorbing aluminum atom (with a core hole), only the $s$ and $p$ for the first six oxygen neighbors, and again the $s, p$ and $d$ for the aluminum next neighbor. The similarity between the absorbing Al $3 p$ local DOS and the XANES spectra shows that the later is a good probe of the former. In the pre-edge region, the role of the absorbing $\mathrm{Al}$ atom is clear as the pre-edge position coincides with its $3 s$ projected DOS. In the case of beryl, a certain proportion of absorbing $\mathrm{Al} 3 d$ states 

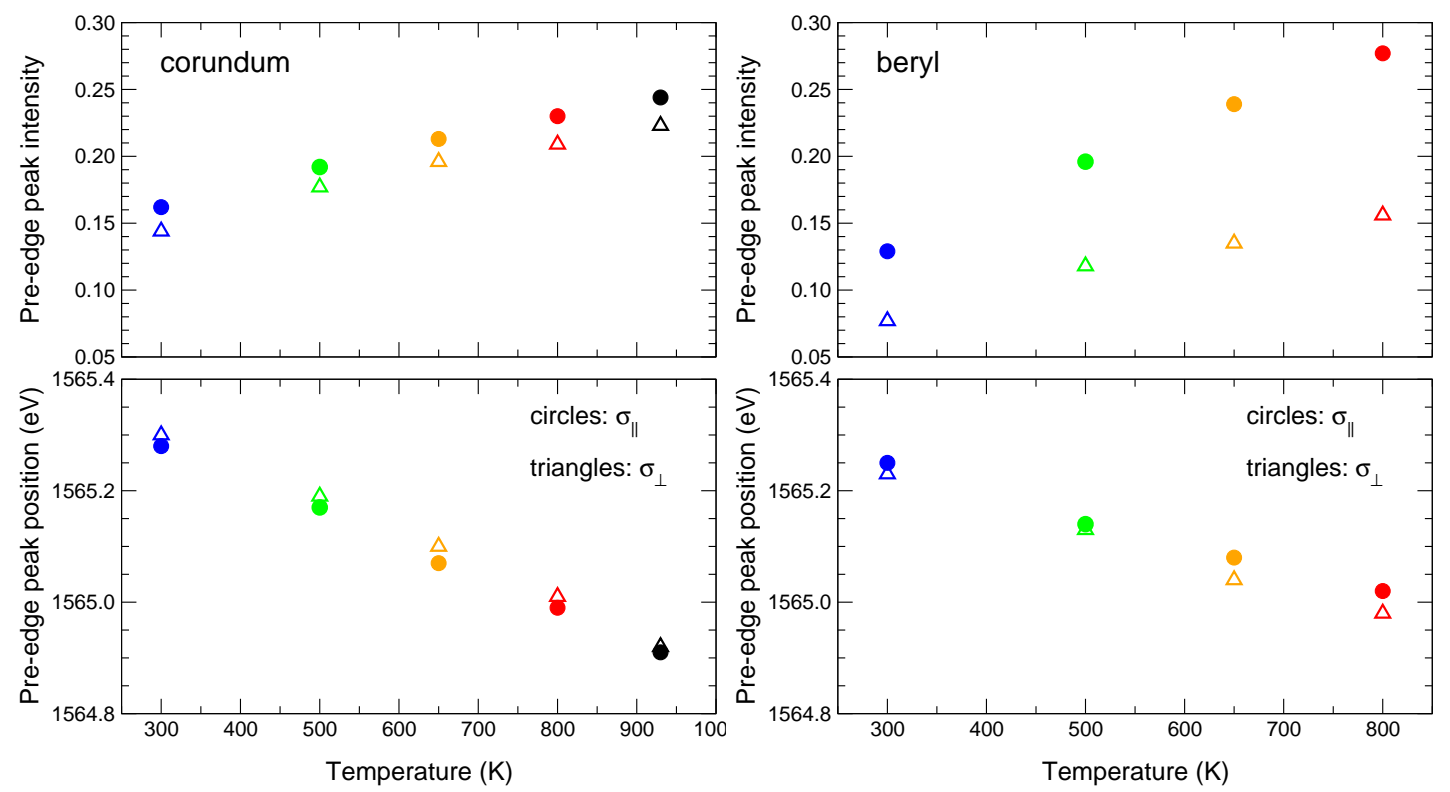

FIG. 4. Pre-edge intensity and energy position for both orientations and for both samples as a function of temperature. The $y$ axis units of the upper panels are consistent with those of Fig. 2

is also present in the pre-edge region. However, the $3 d$ states do not contribute to the absorption coefficient via electric quadrupole $(1 s \rightarrow 3 d)$ transitions. Indeed the calculated electric quadrupole transitions are negligible in the whole XANES region, since they are found to be lower than $0.2510^{-4}$ in the normalized units used in the

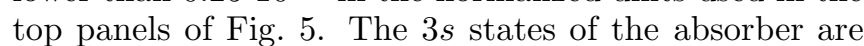
hybridized with both $s$ and $p$ empty states of the oxygen neighbors while the aluminum next neighbors do not contribute to the pre-edge since their DOS are at higher energies. The presence of the $1 s$ core hole on the absorbing atom shifts the first empty states to the bottom of the conduction band and leads to the pre-edge and first peaks of XANES. Non-excited aluminum atoms do not contribute to the pre-edge feature of the spectrum.

\section{B. Theoretical XANES spectra: vibration modeling}

The DOS analysis indicates that the modeling of the experimental Al $K$-edge XANES spectra has to allow transitions toward the $3 s$ states of the absorbing Al. Both method 1 and method 2 presented in section IB are able to achieve this goal. Figure 6 displays the theoretical spectra obtained by the three methods described in section $\amalg B$ together with the spectra calculated with the atoms at their equilibrium positions (labeled 'equil.'). Difference spectra with respect to the 'equil.' reference are also plotted. It should be noted that the 'equil.' spectra do not strictly correspond to the $0 \mathrm{~K}$ case since they do not take the zero point motion of nuclei into account. Experimental spectra measured at $20 \mathrm{~K}$ on the same samples (not shown here) still exhibit the pre-edge peak, even without active phonon modes, and the pre-edge intensity at $20 \mathrm{~K}$ is quite similar to that at $300 \mathrm{~K}$. At room temperature nearly no phonon modes are active, $\stackrel{95,96}{9}$ thus the pre-edge feature observable in the $20 \mathrm{~K}-300 \mathrm{~K}$ range seems to be essentially due to the quantum zero point motion effect.

Corundum $\sigma_{\perp}$ and $\sigma_{\|}$'equil.' spectra reproduce well the overall shape of the experimental spectra, except in the pre-edge-region. Switching on method 1 mostly affects the main edge A intensity and shifts its position by $0.2 \mathrm{eV}$ with respect to 'equil.' spectra. Peaks a and $a^{\prime}$ of the difference spectra are the signature of these effects. Peak $B_{1}$ intensity slightly decreases for both orientations while peak $B_{2}$ is nearly unchanged. Small difference peaks $b_{1}$ and $b_{2}$ confirm these points. A weak pre-edge peak $\mathrm{P}$ seems to appear for both orientations. Although method 1 is not able to grow a well-resolved pre-edge peak, it provides difference spectra that are very similar to the temperature-dependent experimental difference spectra of Fig. III (left panels). On the contrary, method 2 gives rise to a more important pre-edge peak, which is in better agreement with experiment. The difference peak $\mathrm{p}$ shows a really distinct Gaussian-like shape. The rest of the spectrum is nearly unchanged and this is confirmed by the flat difference spectra. Method 3 spectra do not show a pre-edge but have a visible impact on main edge peak $A$, by broadening it, lowering its intensity, and shifting its energy to higher energies by $0.1 \mathrm{eV}$, i.e. in the opposite direction as compared to temperature-dependent experiments. These effects are visible on difference peaks a and a'.

Beryl $\sigma_{\|}$and $\sigma_{\perp}$ 'equil.' spectra also reproduce the experimental spectra quite well, except for some of the peak intensities. While peaks A and C are of similar amplitude 

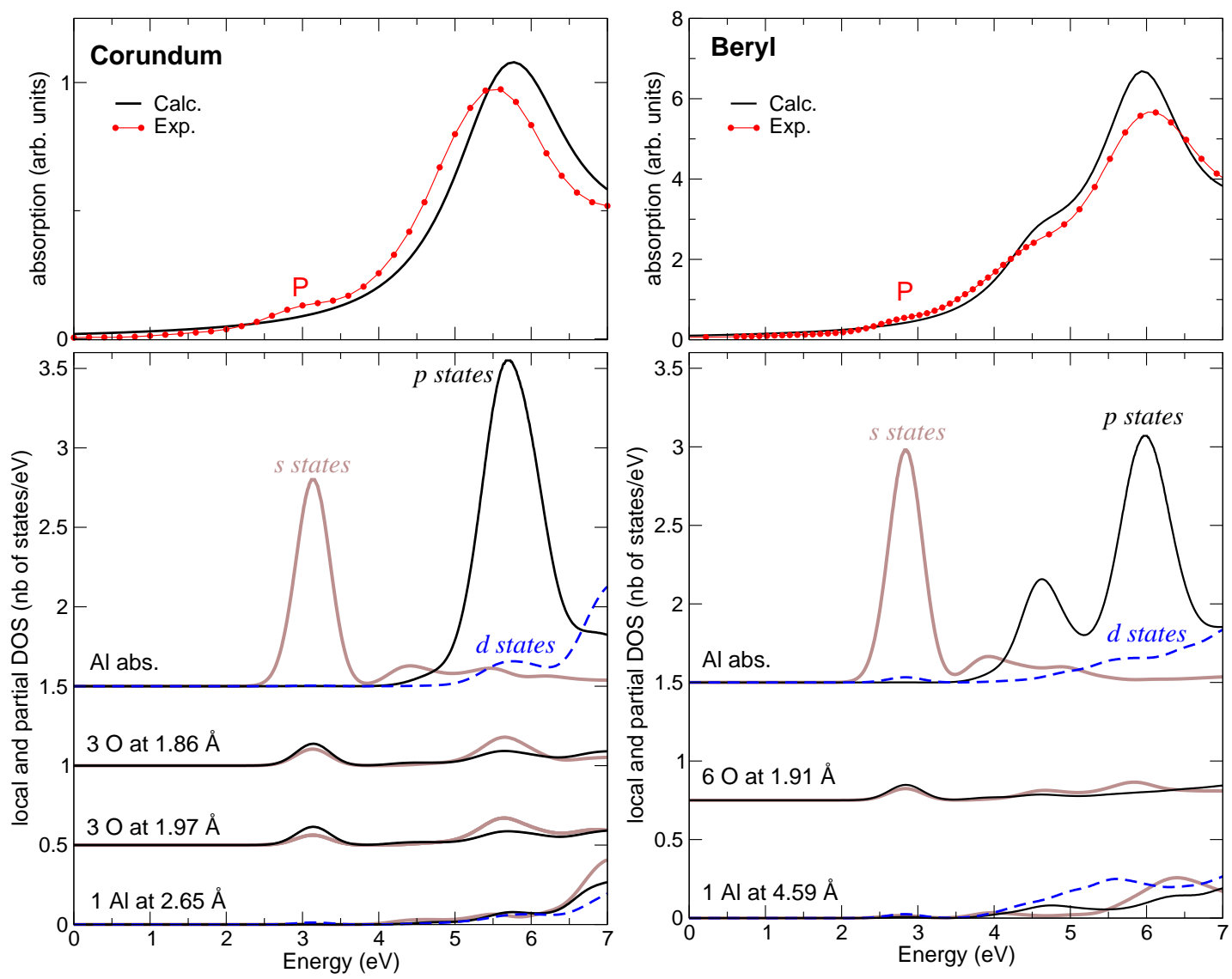

FIG. 5. Top: Calculated and experimental isotropic $\left(2 \sigma_{\perp}+\sigma_{\|}\right) / 3$ spectra at the Al $K$-edge of corundum (left) and beryl(right). Bottom: partial and local density of states of corundum (left) and beryl (right) calculated for the supercell including a $1 s$ core hole. The role of the $\mathrm{Al} 3 \mathrm{~s}$ of the absorbing atom is prominent in the pre-edge region.

in $\sigma_{\|}$experimental spectrum, the intensity of peak A is twice the one of peak $\mathrm{C}$ in the 'equil.' spectrum. On the opposite, peaks B and C have similar intensity in $\sigma_{\perp}$ 'equil.' spectrum while peak B is 1.5 times less intense than peak $\mathrm{C}$ in the $300 \mathrm{~K}$ experimental spectrum. By using method 1 for $\sigma_{\|}$, no pre-edge peak seems to arise, peak A is decreased and shifted to lower energy by $0.2 \mathrm{eV}$, peak $\mathrm{B}$ is also decreased and peak $\mathrm{C}$ remains stable. These effects are also visible through the shape of the difference spectrum. Thus vibrations treated within method 1 are not able to well reproduce the temperature-dependence trend observed in the top-right panel of Fig. 22 In the case of $\sigma_{\perp}$, method 1 leads to theoretical spectrum in better agreement with the x-ray absorption temperaturedependence seen in the bottom-right panel of Fig. 2. Indeed, method 1 enables a well resolved pre-edge peak $\mathrm{P}$ to rise (clearly confirmed by difference peak $\mathrm{p}$ ), and a very weak peak A to appear. It also moves B to lower energy $(0.2 \mathrm{eV})$ and decreases the intensity of peaks $\mathrm{B}$ and C. Here again, method 2 has no influence on peak position, it nearly does not change the amplitude of peaks $\mathrm{A}, \mathrm{B}, \mathrm{C}$ in $\sigma_{\|}$and $\mathrm{B}, \mathrm{C}$ in $\sigma_{\perp}$. However it creates the pre-edge $\mathrm{P}$ at the right energy, but with too high an intensity. The value of the $U$ parameter used in method 2 might be overestimated for beryl and yielded a too pronounced pre-edge peak. Method 3 decreases the amplitude of main peaks. It also shifts peak A of $\sigma_{\|}$and B of $\sigma_{\perp}$ towards higher energies that, as in corundum, is the opposite of what is seen experimentally. A slight broadening appears and is more visible in $\sigma_{\perp}$.

To summarize, method 1 leads to difference spectra that reproduce quite well the temperature-dependent trend observed experimentally in most cases. Nevertheless the impact of vibrations as treated by method 1 is not sufficient to systematically reproduce the experimental pre-edge feature. The $p$-s hybridization induced by this method is not satisfactory enough. In method 1, the vibration modeling results in integration, over a cubic volume around the absorbing atom, of cross sections calculated for absorbing $\mathrm{Al}$ isotropically displaced inside this volume. In other words, this method equally considers the contributions of $\mathrm{Al}$ motion along all directions. Some $\mathrm{Al}$ displacements would be favored by taking into account the phonon modes of the material, what could substantially improve the agreement between theoretical and experimental spectra at least in the pre-edge region. Method 2 spectra all exhibit a well-defined pre-edge peak, corresponding to dipole $1 s-3 s$ transitions, which are al- 

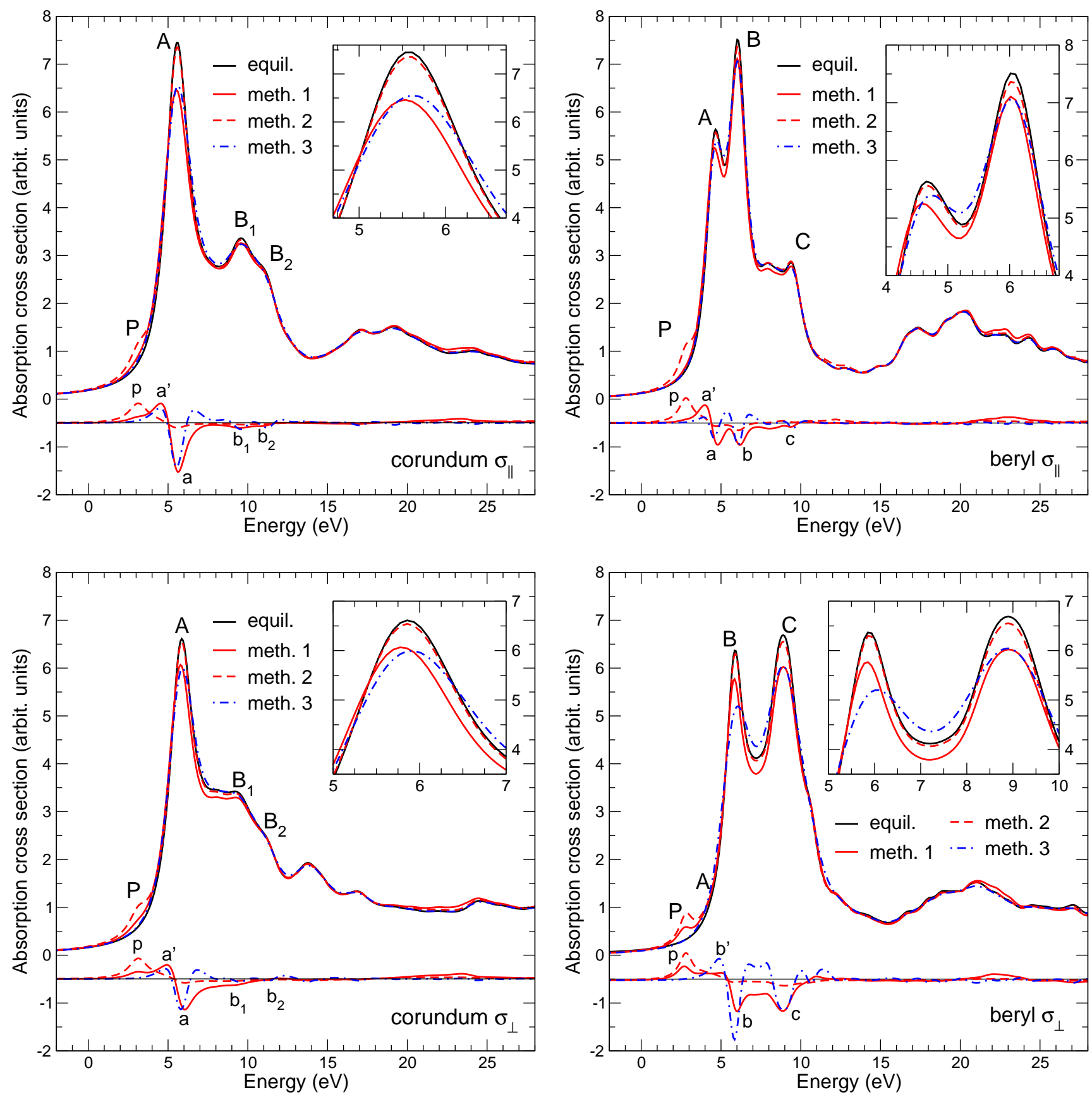

FIG. 6. Calculated $\sigma_{\|}$and $\sigma_{\perp}$ spectra at the $\mathrm{Al} K$ edge of corundum (left) and beryl (right) along with difference of each spectrum with respect to the equil. reference. The 'equil.' spectra correspond to DFT calculation performed with the atoms at the equilibrium positions. The spectra labelled 'meth. 1', 'meth. 2' and 'meth. 3' refer to the three methods of vibration modeling described in section [II. The upper-right inserts use the same legend code and present a zoom on the main peak(s).

lowed by the displacement of the $1 s$ wave-function 1.56 Nevertheless, method 2 has nearly no impact on the peak intensity above the edge, and is unable to produce any shift of the features. This last result was expected because of the use of the crude Born-Oppenheimer (BO) in method 2. Within the crude $\mathrm{BO}$, the transition energies are evaluated for the atoms at their equilibrium positions, hence the spectral features remain at the same energy positions as in 'equil.' spectra. Method 3 spectra, as expected, do not exhibit any pre-edge peak, and present main-edge peaks less intense and shifted in energy. However the energy shift goes the wrong way, i.e. to higher energies. Therefore, a simple convolution of the 'equil.' spectra seems to be inappropriate to account 
for the temperature-dependence of the XANES spectra observed experimentally.

Thermal expansion effects have been evaluated in the case of corundum by performing a XANES calculation for the structural parameters refined at $2170 \mathrm{~K}$ (153K below the melting point) 97 A contraction of the highest energy XANES features has been noticed. Such an effect was expected according to the predictions of the Natoli's rule .98 However, it is contradictory with what has been experimentally observed when temperature is increased (Fig. 2). Therefore, the thermal expansion is unable to explain the temperature-dependence of the Al $K$-edge XANES spectra in corundum and beryl.

The calculations carried out in this study clearly show the crucial role of vibrations in the pre-edge region and partially explain the temperature-dependence observed in the whole XANES region. However, none of the three methods used here is fully satisfactory. A great improvement would be to use PIMD as Schwartz et al. did in the case of two isolated organic molecules. 66 But the computational cost of PIMD simulations in solids, such as corundum or beryl, would be a serious limiting factor. A more reasonable way to account for the thermal fluctuation of XANES at the Al $K$-edge would consist of the generation of atomic configurations of the whole crystal from the dynamical matrix of the system. The temperature-dependent XANES theoretical spectrum would then result in an average of cross-sections calculated for a large number of configurations. An analogous methodology has been successfully employed to account for the temperature-dependence of nuclear magnetic resonance chemical shift in $\mathrm{MgO}^{99}$

\section{CONCLUSION}

In this study, temperature-dependent $\mathrm{Al} K$-edge XANES spectra of corundum and beryl have been presented for temperature ranging from $300 \mathrm{~K}$ to $930 \mathrm{~K}$ for the first time. The XANES spectra were measured on single-crystals with the polarization vector of the x-ray beam parallel and perpendicular to the high symmetry axis of the minerals, allowing the investigation of the temperature dependence of the $\sigma_{\|}$and $\sigma_{\perp}$ components. This series of experiments shows that the pre-edge peak is very sensitive to thermal fluctuations: the pre-edge peak in- tensity grows and its position shifts to lower energy as temperature increases. These variations do not depend much on the x-ray polarization. Thermal fluctuations are also visible above the pre-edge region, through an intensity decrease of the main features and, in the case of corundum, through a slight shift to lower energy of the first main peak.

First-principles DFT calculations have confirmed the $\mathrm{Al} 3 s$ nature of the pre-edge peak in both minerals, and have shown that the introduction of vibrations within the XANES calculation gives rise to a pre-edge peak. Our experimental and theoretical results on the $\mathrm{Al} K$ preedge in corundum and beryl suggest that vibrations (the zero point motion and also temperature) could be able to induce a pre-edge feature at the $\mathrm{Al} K$-edge in other minerals. This conclusion might bring a reinterpretation of the appearance of a pre-edge peak observed at the $\mathrm{Al}$ $K$-edge in zeolites when temperature is increased, which was interpreted as the signature of three-fold coordinated $\mathrm{Al} .43,44,46$

We have used three existing theoretical methods to take nuclear motion into account. These methods have provided useful information to understand the origin of the spectral modifications observed in the temperaturedependent experimental XANES spectra. However, they were found to be inappropriate to fully account for the thermal fluctuation of XANES. This knowledge will be particularly useful for upcoming works, giving insight of what has to be considered in the modeling of temperature-dependent XANES spectra.

\section{ACKNOWLEDGEMENTS}

We are grateful to Steve Collins for showing us the temperature dependence of Ti $K$-edge spectra that he measured on rutile at beamline I16 at Diamond Light Source. This work was granted access to the HPC resources of IDRIS under the allocation 2011-1202 and 2012-100172 made by GENCI (Grand Equipement National de Calcul Intensif). Experiments were performed on the LUCIA beamline at SOLEIL Synchrotron, France (proposal numbers 20100888 and 99110023). We are grateful to the SOLEIL staff for smoothly running the facility. We also acknowledge Pierre Lagarde for fruitful discussions.
* damien.manuel@impmc.upmc.fr

1 C. Brouder, D. Cabaret, A. Juhin, and P. Sainctavit, Phys. Rev. B 81, 115125 (2010)

${ }^{2}$ D. Li, G. M. Bancroft, M. E. Fleet, X. H. Feng, and Y. Pan, Am. Mineral. 80, 432 (1995)

3 D. Li, G. M. Bancroft, M. Kasrai, M. E. Fleet, , X. H. Feng, and K. H. Tan, Am. Mineral. 79, 785 (1994)

4 A. L. Ankudinov and J. J. Rehr, Phys. Scr. T115, 24 (2005)
${ }^{5}$ I. Minkov, F. Gel'mukhanov, R. Friedlein, W. Osikowicz, C. Suess, G. Öhrwall, S. L. Sorensen, S. Braun, R. Murdey, W. R. Salaneck, and H. Ågren, J. Chem. Phys. 121, 5733 (2004)

6 I. Minkov, F. Gel'mukhanov, H. Ågren, R. Friedlein, C. Suess, and W. R. Salaneck, J. Phys. Chem. A 109, 1330 (2005)

7 A. Schöll, Y. Zou, L. Kilian, D. Hübner, D. Gador, C. Jung, S. G. Urquhart, T. Schmidt, R. Fink, and E. Umbach, 
Phys. Rev. Lett. 93, 146406 (2004)

8 S. Carniato, R. Taïeb, E. Kukk, Y. Luo, and B. Brena, J. Chem. Phys. 123, 214301 (2005)

9 V. Ilakovac, S. Carniato, J.-J. Gallet, E. Kukk, D. Horvatić, and A. Ilakovac, Phys. Rev. A 77, 012516 (2008)

10 N. Schmidt, T. Clark, S. G. Urquhart, and R. H. Fink, J. Chem. Phys. 135, 144301 (2011)

11 S. Stranges, R. Richter, and M. Alagia, J. Chem. Phys. 116, 3676 (2002)

12 O. Durmeyer, J.-P. Kappler, E. Beaurepaire, J. M. Heintz, and M. Drillon, J. Phys.: Condens. Matter 2, 6127 (1990)

13 O. Durmeyer, E. Beaurepaire, J.-P. Kappler, C. Brouder, and F. Baudelet, J. Phys.: Condens. Matter 22, 125504 (2010)

14 S. P. Collins and V. E. Dmitrienko(2010), private communication

15 S. Nozawa, T. Iwazumi, and H. Osawa, Phys. Rev. B 72, 121101(R) (2005)

16 B. Ravel, E. A. Stern, Y. Yacobi, and F. Dogan, Jpn. J. Appl. Phys. 32, 782 (1993)

17 B. Ravel and E. A. Stern, Physica B 208\&209, 316 (1995)

18 B. Ravel and E. A. Stern, J. Phys. IV 7, C2 1223 (1997)

19 R. V. Vedrinskii, V. L. Kraizman, A. A. Novakovich, P. V. Demekhin, S. V. Urazhdin, B. Ravel, and E. A. Stern, J. Phys. IV 7, C2 107 (1997)

${ }^{20}$ K. Sato, T. Miyanaga, S. Ikeda, and D. Diop, Phys. Scr. T115, 359 (2005)

${ }^{21}$ T. Hashimoto, A. Yoshiasa, M. Okube, H. Okudera, and A. Nakatsuka, AIP Conf. Proc. 882, 428 (2007)

${ }^{22}$ F. Bridges, C. H. Booth, G. H. Kwei, J. J. Neumeier, and G. A. Sawatzky, Phys. Rev. B 61, R9237 (2000)

${ }^{23}$ Q. Qian, T. A. Tyson, C.-C. Kao, M. Croft, S.-W. Cheong, and M. Greenblatt, Phys. Rev. B 62, 13472 (2000)

24 N. Mannella, A. Rosenhahn, M. Watanabe, B. Sell, A. Nambu, S. Ritchey, E. Arenholz, A. Young, Y. Tomioka, and C. S. Fadley, Phys. Rev. B 71, 125117 (2005)

25 Y. T. Tsai, W. J. Chang, S. W. Huang, J.-Y. Lin, J. Y. Lee, J. M. Chen, K. H. Wu, T. M. Uen, Y. S. Gou, and J. Y. Juang, Physica B 404, 1404 (2009)

${ }^{26}$ R. V. Vedrinskii, E. S. Nazarenko, M. P. Lemeshko, V. Nassif, O. Proux, A. A. Novakovich, and Y. Joly, Phys. Rev. B 73, 134109 (2006)

27 V. A. Shuvaeva, K. Yanagi, K. Yagi, K. Sakaue, and H. Terauchi, J. Synchrotron Rad. 6, 367 (1999)

28 V. A. Shuvaeva, Y. Azuma, K. Yagi, K. Sakaue, and H. Terauchi, J. Synchrotron Rad. 8, 833 (2001)

${ }^{29}$ V. A. Shuvaeva, I. Pirog, Y. Azuma, K. Yagi, K. Sakaue, H. Terauchi, I. P. Raevskii, K. Zhuchkov, and M. Yu Antipin, J. Phys.: Condens. Matter 15, 2413 (2003)

30 V. A. Shuvaeva, Y. Azuma, I. P. Raevski, K. Yagi, K. Sakaue, and H. Terauchi, Ferroelectrics 299, 103 (2004)

31 M. P. Lemeshko, E. S. Nazarenko, A. A. Gonchar, L. A. Reznichenko, T. I. Nedoseykina, A. A. Novakovich, O. Mathon, Y. Joly, and R. V. Vedrinskii, Phys. Rev. B 76, 134106 (2007)

32 A. Deb, J. M. Ralph, E. J. Cairns, and U. Bergmann, Phys. Rev. B 73, 115114 (2006)

33 A. Braun, S. Erat, A. K. Ariffin, R. Manzke, H. Wadati, T. Graule, and L. J. Gauckler, Appl. Phys. Lett. 99, 202112 (2011)

34 J. Herrero-Martín, J. L. García-Muñoz, S. Valencia, C. Frontera, J. Blasco, A. J. Barón-González, G. Subías, R. Abrudan, F. Radu, E. Dudzik, and R. Feyerherm, Phys. Rev. B 84, 115131 (2011)
35 M. Hidaka, N. Tokiwa, M. Oda, J.-Y. Choi, and J. M. Lee, Phase Transit. 76, 905 (2003)

36 B. Poumellec, R. Cortès, E. Loisy, and J. Berthon, phys. stat. sol. (b) 183, 335 (1994)

37 F. Farges, G. E. Brown Jr., A. Navrotsky, H. Gan, and J. J. Rehr, Geoch. Cosmoch. Acta 60, 3055 (1996)

38 O. Dargaud, G. Calas, L. Cormier, L. Galoisy, C. Jousseaume, G. Querel, and M. Newville, J. Am. Ceram. Soc. 93, 342 (2010)

39 D. R. Neuville, D. de Ligny, L. Cormier, G. S. Henderson, J. Roux, A.-M. Flank, and P. Lagarde, Geoch. Cosmoch. Acta 73, 3410 (2009)

40 D. R. Neuville, L. Cormier, D. de Ligny, J. Roux, A.-M. Flank, and P. Lagarde, Am. Mineral. 93, 228 (2008)

41 D. de Ligny, D. R. Neuville, L. Cormier, J. Roux, G. S. Henderson, G. Panczer, S. Shoval, A.-M. Flank, and P. Lagarde, J. Non-Cryst. Sol. 355, 1099 (2009)

42 P. Wernet, D. Nordlund, U. Bergmann, M. Cavalleri, M. Odelius, H. Ogasawara, L. A. Näslund, T. K. Hirsch, L. Ojamäe, P. Glatzel, L. G. M. Pettersson, and A. Nilsson, Science 304, 995 (2004)

43 J. A. van Bokhoven, A. M. J. van der Eerden, and D. C. Koningsberger, J. Am. Chem. Soc. 125, 7435 (2003)

44 J. A. van Bokhoven, Phys. Scr. T115, 76 (2005)

45 A. Omegna, R. Prins, and J. A. van Bokhoven, J. Phys. Chem. B 109, 9280 (2005)

46 G. Agostini, C. Lamberti, L. Palin, M. Milanesio, N. Danilina, B. Xu, M. Janousch, and J. A. van Bokhoven, J. Am. Chem. Soc. 132, 667 (2010)

47 L. H. Aramburo, E. de Smi, B. Arstad, M. M. van Schooneveld, L. Sommer, A. Juhin, T. Yokosawa, H. W. Zandbergen, U. Olsbye, F. de Groot, and B. M. Weckuysen, Angew. Chem., accepted(2012), DOI:10.1002/anie.201109026 and 10.1002/ange.201109026

48 S. J. A. Figueroa, S. J. Stewart, T. Rueda, A. Hernando, and P. de la Presa, J. Phys. Chem. C 115, 5500 (2011)

49 T. Haupricht, R. Sutarto, M. W. Haverkort, H. Ott, A. Tanaka, H. H. Hsieh, H.-J. Lin, C. T. Chen, Z. Hu, and L. H. Tjeng, Phys. Rev. B 82, 035120 (2010)

50 P. Link, P. Glatzel, K. Kvashnina, R. I. Smith, and U. Ruschewitz, Inorg. Chem. 50, 5587 (2011)

${ }^{51}$ F. D. Vila, J. J. Rehr, H. H. Rossner, and H. J. Krappe, Phys. Rev. B 76, 014301 (2007)

52 F. D. Vila, V. E. Lindahl, and J. J. Rehr, Phys. Rev. B 85, $024303(2012)$

53 T. Fujikawa, J. Phys. Soc. Jpn 65, 87 (1996)

${ }^{54}$ T. Fujikawa, J. Phys. Soc. Jpn 68, 2444 (1999)

${ }^{55}$ H. Arai, N. Ueno, and T. Fujikawa, AIP Conf. Proc. 882, $108(2007)$

56 D. Cabaret and C. Brouder, J. Phys.: Conf. Ser. 190, 012003 (2009)

57 H. Köppel, F. X. Gadea, G. Klatt, J. Schirmer, and L. S. Cederbaum, J. Chem. Phys. 106, 4415 (1997)

58 B. Kempgens, B. S. Itchkawitz, J. Feldhaus, A. M. Bradshaw, H. Köppel, M. Döscher, F. X. Gadea, and L. S. Cederbaum, Chem. Phys. Lett. 277, 436 (1997)

59 A. B. Trofimov, T. E. Moskovskaya, E. V. Gromov, H. Köppel, and J. Schirmer, Phys. Rev. A 64, 022504 (2001)

60 A. B. Trofimov, E. V. Gromov, H. Köppel, J. Schirmer, K. C. Prince, R. Richter, M. de Simone, and M. Coreno, J. Phys. B: At. Mol. Opt. Phys. 36, 3805 (2003)

61 D. Duflot, S. Zeggari, and J.-P. Flament, Chem. Phys. 327, 518 (2006) 
62 D. Duflot, J.-P. Flament, A. Giuliani, J. Heinesch, and M.J. Hubin-Franskin, Int. J. Mass Spectrom. 277, 70 (2009)

${ }^{63}$ C. Kolczewski, R. Püttner, O. Plashkevych, H. Ågren, V. Staemmler, M. Martins, G. Snell, A. S. Schlachter, M. Sant'Anna, G. Kaindl, and L. G. M. Pettersson, J. Chem. Phys. 115, 6426 (2001)

${ }^{64}$ G. Herzberg and E. Teller, Z. Physik. Chem. B 21, 410 (1933)

65 J. S. Uejio, C. P. Schwartz, R. J. Saykally, and D. Prendergast, Chem. Phys. Lett. 467, 195 (2008)

66 C. P. Schwartz, J. S. Uejio, R. J. Saykally, and D. Prendergast, J. Chem. Phys. 130, 184109 (2009)

67 C. P. Schwartz, R. J. Saykally, and D. Prendergast, J. Chem. Phys. 133, 044507 (2010)

68 S. Fatehi, C. P. Schwartz, R. J. Saykally, and D. Prendergast, J. Chem. Phys. 132, 094302 (2010)

69 F. Della Sala, R. Rousseau, A. Görling, and D. Marx, Phys. Rev. Lett. 92, 183401 (2004)

70 A. Kaczmarek, M. Shiga, and D. Marx, J. Phys. Chem. A 113, 1985 (2009)

71 S. Mazevet and G. Zérah, Phys. Rev. Lett. 101, 155001 (2008)

72 V. Recoules and S. Mazevet, Phys. Rev. B 80, 064110 (2009)

73 O. Peyrusse, High Energy Density Phys. 6, 357 (2010)

74 A. Benuzzi-Mounaix, F. Dorchies, V. Recoules, F. Festa, O. Peyrusse, A. Levy, A. Ravasio, T. Hall, M. Koenig, N. Amadou, E. Brambrink, and S. Mazevet, Phys. Rev. Lett. 107, 165006 (2011)

75 A. Lévy, F. Dorchies, A. Benuzzi-Mounaix, A. Ravasio, F. Festa, V. Recoules, O. Peyrusse, N. Amadou, E. Brambrink, T. Hall, M. Koenig, and S. Mazevet, Phys. Rev. Lett. 108, 055002 (2012)

76 A.-M. Flank, G. Cauchon, P. Lagarde, S. Bac, M. Janousch, R. Wetter, J.-M. Dubuisson, M. Idir, F. Langlois, T. Moreno, and D. Vantelon, Nucl. Instrum. Meth. B 246, 269 (2006)

77 J. A. Bearden and A. F. Burr, Rev. Mod. Phys. 39, 125 (1967)

78 R. E. Newnham and Y. M. de Haan, Z. Kristallogr. 117, $235(1962)$

79 R. M. Hazen, A. Y. Au, and L. W. Finger, Am. Mineral. 71, $977(1986)$

80 C. Brouder, J. Phys.: Condens. Matter 2, 701 (1990)

81 J. Goulon, C. Goulon-Ginet, R. Cortes, and J. M. Dubois, J. Physique 43, 539 (1982)
82 D. Haskel, "FLUO: Correcting XANES for selfabsorption in fluorescence measurements," (1999), http://www.aps.anl.gov/xfd/people/haskel/fluo.html

${ }^{83}$ E. B. Saloman, J. H. Hubbell, and J. H. Scofield, Atom. Data Nucl. Data Tables 38, 1 (1988)

${ }^{84}$ P. Giannozzi, S. Baroni, N. Bonini, M. Calandra, R. Car, C. Cavazzoni, D. Ceresoli, G. L. Chiarotti, M. Cococcioni, I. Dabo, A. Dal Corso, S. Fabris, G. Fratesi, S. de Gironcoli, R. Gebauer, U. Gerstmann, C. Gougoussis, A. Kokalj, M. Lazzeri, L. Martin-Samos, N. Marzari, F. Mauri, R. Mazzarello, S. Paolini, A. Pasquarello, L. Paulatto, C. Sbraccia, S. Scandolo, G. Sclauzero, A. P. Seitsonen, A. Smogunov, P. Umari, and R. M. Wentzcovitch, J. Phys.: Condens. Mat. 21, 395502 (2009)

85 N. Troullier and J. L. Martins, Phys. Rev. B 43, 1993 (1991)

86 J. P. Perdew, K. Burke, and M. Ernzerhof, Phys. Rev. Lett. 77, 3865 (1996)

87 C. Gougoussis, M. Calandra, A. P. Seitsonen, and F. Mauri, Phys. Rev. B 80, 075102 (2009)

88 M. Taillefumier, D. Cabaret, A.-M. Flank, and F. Mauri, Phys. Rev. B 66, 195107 (2002)

89 P. E. Blöchl, Phys. Rev. B 50, 17953 (1994)

90 M. Abramowitz and I. Stegun, Handbook of mathematical functions with formulas, graphs and mathematical tables (Dover Publications, 1964)

91 P. Thompson and I. G. Wood, J. Appl. Crystallogr. 16, 458 (1983)

92 D. Cabaret, E. Gaudry, M. Taillefumier, P. Sainctavit, and F. Mauri, Phys. Scr. T115, 131 (2005)

93 P. Carra, H. Koenig, B. T. Thole, and M. Altarelli, PhysicaB 192, 182 (1993)

94 M. Wojdyr, J. Appl. Cryst. 43, 1126 (2010)

95 H. Bialas and H. J. Stoltz, Z. Phys. B 21, 319 (1975)

96 M. Prencipe, Y. Noel, B. Civalleri, C. Roetti, and R. Doveri, Phys. Chem. Minerals 33, 519 (2006)

97 N. Ishizawa, T. Miyata, I. Minato, F. Marumo, and S. Iwai, Acta Cryst. B 36, 228 (1980)

98 C. R. Natoli, "Near-edge absorption structure in the framework of the multiple scattering model. potential resonance or barrier effects?." in EXAFS and Near Edge Structure, Springer Series in Chemical Physics, Vol. 27 (Berlin: Springer, 1983) pp. 43-56

99 S. Rossano, F. Mauri, C. J. Pickard, and I. Farnan, J. Phys. Chem. B 109, 7245 (2005) 